\title{
ВЛИЯНИЕ COVID-19 НА РОССИЙСКУЮ ЭКОНОМИКУ: МЕТОДОЛОГИЧЕСКИЕ ПОДХОДЫ К ОЦЕНКЕ НА ОСНОВЕ МЕЖОТРАСЛЕВОГО БАЛАНСА*
}

\author{
А.А. Быков, В.А. Пархименко, С.А. Толкачев**
}

Предпринята попытка оценки влияния пандемии COVID-19 на состояние экономики Российской Федерации исходя из предполагаемых значений падения внутреннего спроса по каждой из 98 отраслей (продуктов), выделенных в системе таблиц «затраты - выпуск». Анализ проводился в разрезе выделения двух ключевых факторов изменений спроса: карантинные меры, напрямую ограничивающие внутренний спрос, а также мировые цены на нефть, ведущие к сокращению экспортных доходов экономики и последующему сокращению спроса на товары и услуги на внутреннем рынке. Полученные количественные оценки представлены в разрезе отдельных отраслей, секторов и российской экономики в целом. В качестве вывода обосновывается оценка совместного влияния эффектов карантина и снижения экспорта нефти на уровне около 5\% ВВП.

Ключевые слова: анализ «затраты - выпуск», карантин, экономический рост, экономические последствия пандемии COVID-19, Российская Федерация.

JEL-классификация: C67, D57, E17, O41.

DOI: $10.46782 / 1818-4510-2020-2-25-37$

Материал поступил 10.06.2020 2.

\section{Постановка проблемы}

Спустя полгода после начала пандемии COVID-19 оценке ее экономических последствий посвящено множество исследований. Однако события развиваются быстрее, чем публикуются научные работы, поэтому статей в научных рецензируемых журналах на эту тему пока мало. В рамках данной проблематики анализируются совершенно разные экономические и демографические параметры: например, зависимость уровня смертности населения от мобильности, энергообеспеченности и наличия страховок (Chen, Igan, Pierri, Presbitero, 2020); фор-

* Статья выполнена в рамках совместного научного проекта по гранту БРФФИ-РФФИ «Оценка макроэкономической эффективности интеграции отраслей обрабатывающей промышленности России и Республики Беларусь в глобальные цепочки стоимости», номер проекта РФФИ 20-510-00026 Бел; номер госрегистрации в Республике Беларусь № 20200909 . мируются прогнозы ВВП в зависимости от силы ограничений во время карантина ${ }^{1}$. В отмеченной статье на примере выборки из 30 стран показано, что в краткосрочном периоде жесткость ограничений напрямую влияет на ожидаемый спад ВВП. Пока не определена общепринятая методология оценки экономических последствий пандемии. Ее распространение в популяции описывается вероятностными причинно-следственными моделями (Friston, Parr, Zeidman, Razi, Flandin, Daunizeau, Hulme, Billig, Litvak, Moran, Price, Lambert, 2020), однако экономические расчеты не обязательно должны осуществляться по схожей методологии.

На данном этапе развития событий, когда пандемия далека от завершения, и количество возможных сценариев кризиса в ми-

1 Sweden: Will COVID-19 Economics be Different? 2020. URL: https://www.imf.org/en/News/Articles/2020/06/ 01/na060120-sweden-will-covid-19-economics-be-different

** Быков Алексей Александрович (aliaksei.bykau@yandex.ru), доктор экономических наук, профессор, Белорусский государственный экономический университет (г. Минск, Беларусь);

Пархименко Владимир Анатольевич (parkhimenko@bsuir.by), кандидат экономических наук, доцент, Белорусский государственный университет информатики и радиоэлектроники (г. Минск, Беларусь);

Толкачев Сергей Александрович (tsa2000@mail.ru), доктор экономических наук, профессор, Финансовый университет при Правительстве Российской Федерации, (г. Москва, Россия). 
ровой экономике велико, слишком сложно математически точно описать динамику экономических параметров. Для описания реакции национальной экономики на последствия пандемии нами предлагается использовать более простые подходы к прогнозированию, основанные на экспертных оценках изменения спроса, зная которые можно рассчитать изменения ВВП по данным межотраслевого баланса. Изменения спроса в течение года можно приблизительно оценить уже сейчас по статистике перемещений людей и некоторым данным за I квартал 2020 г. Также существует возможность прогнозирования доходов от экспорта исходя из ожидаемой динамики мировых товарных рынков.

Авторы представили свой вариант оценки влияния пандемии COVID-19 на состояние экономики Российской Федерации исходя из оценок падения внутреннего спроса по каждой из 98 отраслей (продуктов), выделенных в системе таблиц «затраты - выпуск» за 2016 г. (последней из доступных на момент написания статьи) ${ }^{2}$. При этом использовалась традиционная для анализа «затраты-выпуск» предпосылка о том, что технологические коэффициенты (явным образом не выделяемые в российской статистике) в период 2016-2020 гг. не претерпели существенных изменений.

Применительно к российской экономике, рассмотрены два ключевых фактора изменений спроса: карантин, ограничивающий физические перемещения людей и их доходы; мировые цены на нефть, снижение которых приводит к сокращению доходов секторов экономики и последующему сокращению спроса на товары и услуги на внутреннем рынке.

В целом оценка и моделирование экономического роста на основе изменений спроса характерны для неокейнсианской экономической школы. Этот подход в совокупности с методологией межотраслевого баланса был применен для анализа факторов экономического роста белорусской экономики (Быков, Шаблинская, 2020). Важным параметром большинства неокейнсианских моделей является обменный курс валюты, через который осуществляет-

${ }^{2}$ Национальные счета. Федеральная служба государственной статистики. URL: https://gks.ru/accounts ся взаимосвязь изменений экспорта с изменениями внутреннего спроса. В работе мы сознательно отказались от использования данного параметра в модели, о чем более подробно будет сказано ниже.

\section{Методология оценки влияния конечного спроса на макроэкономические показатели посредством модели межотраслевого баланса}

Классическая модель межотраслевого баланса (МОБ) Леонтьева (Leontief, 1986) в матричной форме может быть представлена как:

$$
A X+Y=X
$$

где $A$ - матрица технологических коэффициентов или прямых затрат, в которой элемент $a_{i j}$ показывает, сколько продукции $i$-й отрасли поставляется в $j$-ю отрасль для производства единицы продукции $j$-й отрасли. В Российской Федерации матрица прямых затрат не приводится в официальной статистике, однако может быть посчитана по данным симметричной таблицы «затраты - выпуск», ее размерность при этом будет равна $98 \times 98$;

$X$ - вектор-столбец выпуска товаров и услуг в основных ценах. В случае российской статистики его размерность $98 \times 1$;

$Y$ - вектор-столбец конечного спроса (конечного использования) с размерностью, равной размерности вектора-столбца $X$. В конечный спрос включаются конечное потребление (домохозяйств, государственных учреждений и т. д.), накопление (основного капитала и оборотных средств) и экспорт. В российской системе таблиц «затраты - выпуск» указанные компоненты представлены и доступны для анализа, как отдельно, так и в виде итоговых (суммарных) значений.

Уравнение (1) показывает очевидный экономический факт: весь выпуск товаров и услуг за определенный период идет на промежуточное и конечное использование.

При этом в матрице $A$ отображены, по сути, производственные функции («рецептура» производства) каждой из выделяемых отраслей и, соответственно, производственные (технологические) цепочки в рамках национальной экономики. Предполагается, что 
значения элементов матрицы заданы и относительно устойчивы во времени (Wood, 2011), что позволяет использовать ее значения за прошлые периоды для прогнозирования будущих состояний экономики.

Выпуск в модели Леонтьева является эндогенной величиной и находится для заданных матрицы $A$ и конечного спроса $Y$ (экзогенная величина, определяемая внешними факторами).

Введение в рассмотрение импортных товаров и услуг, которые вместе с отечественными товарами и услугами идут на промежуточное и конечное использование, требует некоторого изменения базовой модели. На практике статистические органы предоставляют необходимые для этого исходные данные (детальные таблицы использования отечественных и импортных товаров и услуг).

В литературе существуют разные подходы к учету импорта в рамках межотраслевого баланса (Точицкая, 2019; Кравцов, Антаневич, 2015; Готовский, 2019). Представленный ниже подход в общем и целом соответствует традиционной методологии анализа «затраты-выпуск» и близок подходу, рассмотренному в публикациях (Chen, Cheng, Fung, Lau, 2004), а также (Koopman, Wang, Wei, 2008).

Для учета импортных потоков товаров и услуг предлагается модифицировать уравнение (1) следующим образом:

1) разбить матрицу прямых затрат на две - матрицу прямых затрат на промежуточное потребление отечественных товаров и услуг и матрицу прямых затрат на промежуточное потребление импортных товаров и услуг (исходя из таблицы использования отечественных товаров и услуг и таблицы использования импортных товаров и услуг):

$$
A=A_{\text {отеч }}+A_{\text {имп }} \text {; }
$$

2) разбить вектор-столбец конечного использования (включая экспорт) на два конечное использование отечественных товаров и услуг и конечное использование импортных товаров и услуг (исходя из данных тех же таблиц):

$$
Y=Y_{\text {отеч }}+Y_{\text {имп }}
$$

3) учесть, что в рамках конечного использования потребляется и произведен- ный внутри страны выпуск $(X)$, и импортируемые товары и услуги $(I)$. Тогда уравнение (1) будет представлено в виде:

$$
\left(A_{\text {отеч }}+A_{\text {имп }}\right) \cdot X+\left(Y_{\text {отеч }}+Y_{\text {имп }}\right)=X+I,
$$

где $A_{\text {отеч }}-$ матрица прямых затрат, построенная по данным промежуточного потребления отечественных товаров и услуг (размерностью $98 \times 98$ для России);

$A_{\text {имп }}$ - матрица прямых затрат, построенная по данным промежуточного потребления импортных товаров и услуг (размерностью $98 \times 98$ для России);

$$
Y_{\text {отеч }} \text { - вектор-столбец, содержащий }
$$
данные по конечному использованию (включая экспорт) отечественных товаров и услуг (размерностью $98 \times 1$ для России);

$Y_{\text {имп }}$ - вектор-столбец, описывающий конечное использование (включая экспорт) импортных товаров и услуг (размерностью 98 х 1 для России);

$X$ - вектор-столбец, описывающий произведенный внутри страны общий объем выпуска товаров и услуг в основных ценах (размерностью $98 \times 1$ ). Полностью соответствует вектору-столбцу $X$ в уравнении (1);

$I$ - вектор-столбец, описывающий общий объем импортируемых товаров и услуг (размерностью $98 \times 1$ ).

Очевидно, что импортные товары и услуги потребляются в рамках промежуточного потребления (при этом они линейно возрастают с ростом объемов выпуска внутри страны) и в рамках конечного использования (предполагается, что они зависят не от объема выпуска, а от экзогенных факторов - предпочтений домашних хозяйств и т.д.), то есть можно представить общий объем импорта как:

$$
I=A_{\text {имп }} \cdot X+Y_{\text {имп }}
$$

Если подставить (5) в (4) и решить (4) относительно $X$, получим следующее выражение:

$$
X=\left(E-A_{\text {отеч }}\right)^{-1} \cdot Y_{\text {отеч }},
$$

где $E$ - единичная матрица соответствующей размерности $98 \times 98$. 
Матрица $\left(E-A_{\text {отеч}}\right)^{-1}$ в $(6)$ является матрицей полных затрат отечественных товаров и услуг на единицу (1 руб.) конечного спроса. В российской системе таблиц «затраты - выпуск» это матрица дана в таблице «Коэффициенты полных затрат отечественной продукции», однако значения приведены на 1000 руб. продукции.

Учитывая изначальную заданность и относительную стабильность матрицы технологических коэффициентов (в данном случае - матрицы $\left.A_{\text {отеч}}\right)$, выпуск зависит исключительно от конечного спроса на отечественные товары и услуги (в том числе со стороны зарубежных потребителей) и, таким образом, изменение в выпуске можно записать в виде:

$$
\Delta X=\left(E-A_{\text {отеч }}\right)^{-1} \cdot \Delta Y_{\text {отеч }}
$$

Здесь и далее $\Delta$ с соответствующим вектором-столбцом обозначает вектор-столбец, в котором отражены изменения каждого из элементов вектора-столбца по отношению к их базовым значениям, т. е. например, $\Delta X=X^{\prime}-X$, где $X$ и $X^{\prime}-$ векторыстолбцы выпуска до и после изменения конечного спроса на отечественные товары и услуги.

При этом ввиду линейности модели равномерное увеличение конечного спроса по всем отраслям (по всем элементам векторастолбца ) приведет к пропорциональному изменению выпуска по каждой отрасли (т. е. каждого элемента вектора-столбца $X$ ). В этом контексте сильная сторона модели межотраслевого баланса проявляется только в том случае, когда конечный спрос по разным отраслям испытывает различные изменения (меняется структура конечного спроса) и может быть зафиксирована реакция различных производственных цепочек, образующих национальную экономическую систему.

Особый интерес представляет модификация (7), описывающая ситуацию, когда прирост конечного спроса на отечественные товары и услуги происходит исключительно за счет изменения внутреннего спроса, при неизменном экспорте. Для этого воспользуемся подстановкой:

$$
Y_{\text {отеч }}=D_{\text {отеч }}+\operatorname{Exp}_{\text {отеч' }}
$$

где $D_{\text {отеч }}-$ вектор-столбец внутреннего конечного спроса на отечественные товары и услуги (конечное потребление и накопление);

$\operatorname{Exp}_{\text {отеч }}$ - вектор-столбец экспорта отечественных товаров и услуг.

Исходя из (8), можно переписать (7) в общем случае в виде двух слагаемых: первое описывает изменения выпуска за счет изменения спроса на отечественные товары и услуги внутри страны, а второе - за счет изменения экспорта отечественных товаров и услуг:

$$
\begin{aligned}
& \Delta X=\left(E-A_{\text {отеч }}\right)^{-1} \cdot \Delta D_{\text {отеч }}+ \\
& +\left(E-A_{\text {отеч }}\right)^{-1} \cdot \Delta \operatorname{Exp}_{\text {отеч }}
\end{aligned} .
$$

Знание прогнозного значения объемов выпуска позволяет отыскать и другие макроэкономические показатели. Общий размер валовой добавленной стоимости по отраслям может быть описан следующей системой уравнений:

$$
v a_{j}=x_{j}\left(1-\sum_{i=1}^{n} a_{i j}\right) \text { для } j=\overline{1, n},
$$

где $v a_{j}-$ валовая добавленная стоимость $j$-й отрасли;

$x_{j}-$ выпуск $j$-й отрасли, т. е. $j$-й элемент вектора-столбца $X$;

$a_{i j}-$ технологический коэффициент, то есть $i j$-й элемент матрицы $A$.

В рамках модели Леонтьева валовая добавленная стоимость линейно зависит от выпуска отрасли при условии неизменности технологических коэффициентов: абсолютные значения валовой добавленной стоимости увеличиваются или снижаются в зависимости от объема выпуска, доля же этой стоимости в выпуске остается постоянной.

Обозначим за $v_{j}$ долю валовой добавленной стоимости в выпуске $j$-й отрасли и определим ее как

$$
v_{j}=\frac{v a_{j}}{x_{j}} .
$$

Пусть $V$ - вектор-столбец, содержащий в качестве элементов $v_{j}(j=\overline{1, n})$. Тогда систему уравнений (8) можно будет записать в матричной форме, используя произведение Адамара ${ }^{3}$ : 


$$
V A=V \circ X
$$

Так как модель Леонтьева линейная, то при прочих равных рост выпуска отечественных товаров и услуг приведет к пропорциональному росту валовой добавленной стоимости $V A$ :

$$
\triangle V A=V \circ \Delta X .
$$

Подставляя в уравнение (13) вместо вектора-столбца $V$ векторы-столбцы, характеризующие доли отдельных элементов добавленной стоимости, например, долю зарплаты в выпуске, получим изменение соответствующего элемента в абсолютном выражении.

В (13) вектор-столбец $\Delta V A$ описывает совокупность изменений размера добавленной стоимости по каждой из отраслей (продукту). Для оценки изменения размера валового внутреннего продукта (это уже будет не вектор-столбец, а число, поэтому в формуле ниже используются строчные буквы вместо прописных) можно обратиться к формуле, которая учитывает чистые налоги на продукты в конечном использовании:

$$
\Delta g d p=\sum_{j=1}^{n} \Delta x_{j} v_{j}+t^{X} \cdot \Delta \exp +t^{D} \cdot \Delta d
$$

где $\Delta \exp$ - прогнозируемое изменение итоговой суммы валового экспорта отечественных и импортных товаров и услуг;

$\Delta d$ - прогнозируемое изменение итоговой суммы конечного потребления и валового накопления отечественных и импортных товаров и услуг;

$t^{X}$ - отношение чистых налогов на продукты в экспорте к валовому экспорту;

$t^{D}$ - отношение чистых налогов на продукты в конечном потреблении и валовом накоплении к сумме конечного потребления и валового накопления;

$x_{j}, v_{j}-$ элементы векторов-столбцов $X$, $V$ соответственно.

В последующих расчетах нами также используются показатели добавленной стоимости национального происхождения / импортоемкости в расчете на единицу конечного использования товаров и услуг. Для их расчета обозначим $A_{\text {ммп }} \cdot\left(E-A_{\text {отеч }}\right)^{-1}$ как матрицу $C$, которая может пониматься как матрица полной импортоемкости или как совокупность полных затрат на импор- тные товары и услуги, необходимые для производства единицы продукции, при изменении конечного использования отечественных товаров и услуг на единицу (1 руб.). В частности, конкретный элемент матрицы $c_{i j}$ показывает, насколько увеличится потребность в импорте по $i$-й отрасли при увеличении на единицу конечного использования отечественных товаров и услуг $j$-отрасли.

Таким образом, импортная составляющая (прямая и косвенная) в $j$-м выпуске будет равна:

$$
c_{j}=\sum_{i=1}^{n} c_{i j}
$$

А добавленная стоимость национального происхождения по $j$-й отрасли (продукту) на единицу валового выпуска, соответственно, может быть найдена как разность $1-c_{j}$.

С точки зрения базовой логики модели Леонтьева импортоемкость и добавленная стоимость национального происхождения на единицу валового выпуска являются величинами неизменными и задаваемыми «рецептурой» производства по $j$-й отрасли.

При этом сумма валового экспорта товаров и услуг по всем отраслям $\left(\exp _{\text {отеч } j}, j=\overline{1, n}\right)$, взвешенная с учетом коэффициентов добавленной стоимости конечных продуктов $\left(1-c_{j}\right)$, дает нам общую величину добавленной стоимости национального происхождения в экспорте (VAX). Сумма конечного использования отечественных товаров и услуг по всем отраслям $\left(d_{\text {отеч } j}, j=\overline{1, n}\right)$, взвешенная с учетом коэффициентов добавленной стоимости конечных продуктов $\left(1-c_{j}\right)$, показывает величину добавленной стоимости национального происхождения, созданную за счет продаж на внутреннем рынке (VAD).

Обобщая, можно свести предлагаемые расчетные формулы изменения важных макроэкономических показателей под воздействием изменений в конечном спросе и/или отдельных его компонентов в таблицу.

Предложенная методология позволяет на основе оценок (прогноза) значений конечного спроса и/или его компонентов (в первую очередь, внутренний и внешний спрос) осуществлять оценку (прогнозиро- 
Основные формулы для оценки влияния изменений в конечном спросе и отдельных его компонентов на макроэкономические показатели национальной экономики

\begin{tabular}{|c|c|}
\hline Макроэкономический показатель & Формула для расчета \\
\hline $\begin{array}{l}\text { Изменения выпуска товаров и услуг по каждой из отраслей } \\
\text { («продуктов») }\end{array}$ & $\Delta X=\left(E-A_{\text {отеч}}\right)^{-1} \cdot \Delta Y_{\text {отеч }}$ \\
\hline $\begin{array}{l}\text { Изменение валовой добавленной стоимости по каждой из } \\
\text { отраслей («продуктов») }\end{array}$ & $\Delta V A=V \circ\left(E-A_{\text {отеч}}\right)^{-1} \cdot \Delta Y_{\text {отеч }}$ \\
\hline Изменение ВВП & $\Delta g d p=\sum_{j=1}^{n} \Delta x_{i j} y+t^{X} \cdot \Delta \exp +t^{D} \cdot \Delta d$ \\
\hline Импортоемкость (матрица размерностью 98 х 98) & $C=A_{\text {имп }} \cdot\left(E-A_{\text {огеч }}\right)^{-1}$ \\
\hline $\begin{array}{l}\text { Добавленная стоимость национального происхождения в } \\
\text { экспорте }\end{array}$ & $V A X=\sum_{j=1}^{n} \exp _{\text {отеч } j} \cdot\left(1-c_{j}\right)$ \\
\hline $\begin{array}{l}\text { Добавленная стоимость, созданная за счет продаж на } \\
\text { внутреннем рынке }\end{array}$ & $V A D=\sum_{j=1}^{n} d_{\text {огеч } j} \cdot\left(1-c_{j}\right)$ \\
\hline
\end{tabular}

Источник. Авторская разработка.

вание) ключевых макроэкономических показателей.

Расчеты проводились в Microsoft Excel по формулам из таблицы. Несмотря на довольно большую размерность исходных матриц $(98 \times 98)$, сами расчеты относительно просты, так как в Excel встроены операторы матричной алгебры: МУМНОЖ(), ТРАНСП(), МОБР().

\section{Оценка влияния карантина на внутренний спрос и ВВП}

Карантин влияет на совокупный спрос на внутреннем рынке через снижение продаж товаров и услуг. Предполагается, что введение карантина ограничит спрос на внутреннем рынке. Изменение спроса касается конечного потребления и валового накопления продуктов на внутреннем рынке. Эти показатели установлены экспертным путем, в среднегодовом измерении. В наибольшей степени снизится спрос на персональные услуги - гостиниц и ресторанов, общественного питания, однако данные отрасли, по нашим расчетам, внесли не самый существенный вклад в прогнозируемое изменение ВВП ввиду их низкой доли в ВВП и относительно слабой связанности с прочими отраслями экономики. Наибольший вклад в изменение ВВП, по нашим оценкам, внесет спрос на продукты, перечисленные на рис. 1.

Наибольшее влияние на ожидаемый экономический спад оказали отрасли, сни- жение спроса на услуги которых оказалось не самым значительным - оптовая и розничная торговля. Это произошло из-за сравнительно высоких долей этих отраслей в ВВП, а также в связи с глубокой интеграцией оптовой и розничной торговли с прочими отраслями российской экономики.

В качестве иллюстрации рассмотрим влияние изменений спроса на услуги розничной торговли на выпуск смежных продуктов, которые используются в промежу-

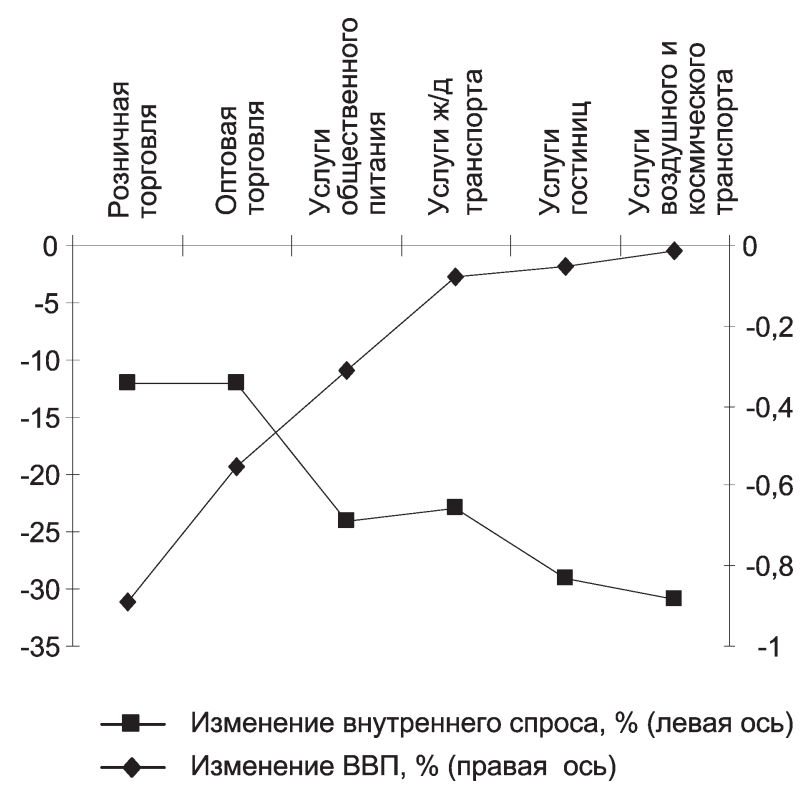

Рис. 1. Изменение внутреннего спроса по некоторым отраслям (экспертные оценки) и расчетное изменение ВВП в 2020 г., \%

Источник. Авторская разработка. 
точном потреблении розничной торговлей. Например, спрос на услуги розничной торговли, согласно экспертной оценке, сократится на 12\%. Модель МОБ показывает, как данный факт отразится на выпуске других продуктов: почти на 3\% снизится выпуск офисного оборудования; на 1\% - печатной продукции и печатных материалов, услуг по аренде; на 0,7\% - производство электроэнергии и услуг финансового посредничества (рис. 2).

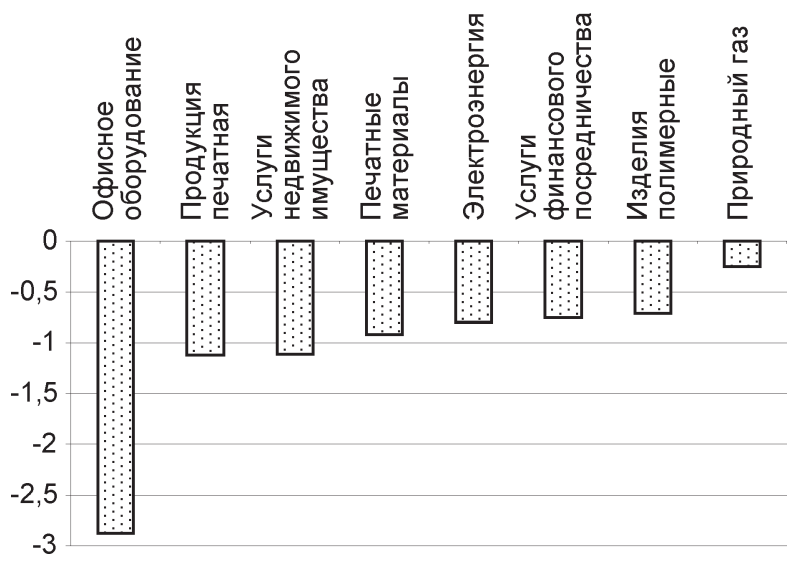

Рис. 2. Реакция объемов выпуска отдельных отраслей на снижение в размере $12 \%$ спроса на услуги розничной торговли, \%

Источник. Авторская разработка.

Общее влияние ожидаемых изменений совокупного спроса на внутреннем рынке на показатели выпуска и ВВП приведены на рис. 3. Более глубокая детализация по

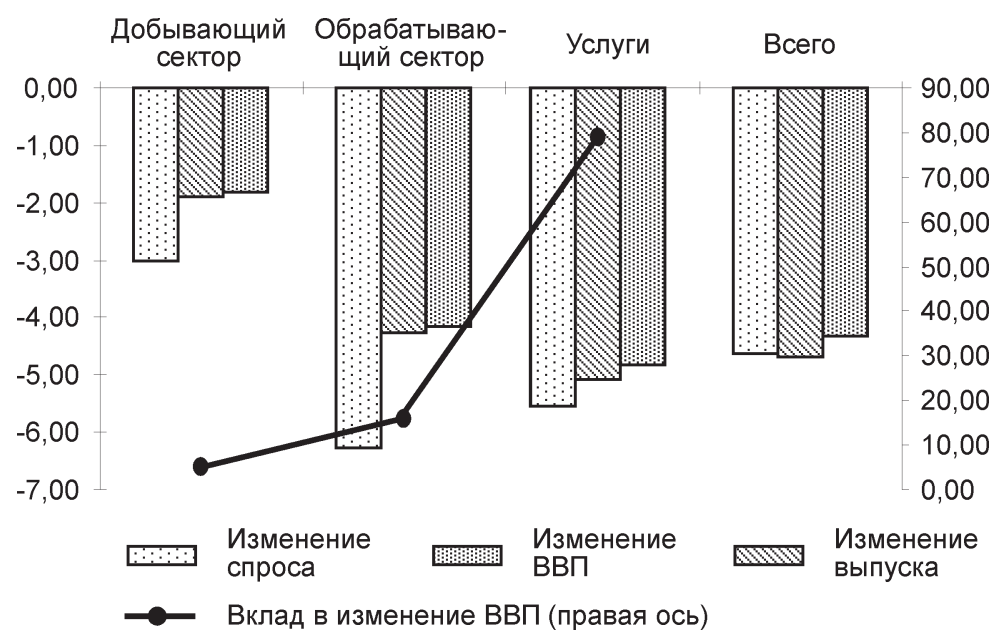

Рис 3. Влияние ожидаемых изменений внутреннего спроса на выпуск и ВВП в 2020 г., \%

Источник. Авторская разработка видам деятельности не показана ввиду широкого перечня продуктов в МОБ - 98 позиций.

Как видно, прогнозируемое снижение внутреннего спроса в годовом измерении составит 4,63\%, что приведет к сокращению выпуска на 4,69\% и снижению ВВП на 4,33\%. Наибольший вклад в снижение ВВП (79\%) обеспечивает сектор услуг. Этот показатель является исключительно результатом карантина и вызван снижением только внутреннего спроса.

\section{Методика расчета влияния экспорта на ВВП}

Влияние экспорта на изменение ВВП обеспечивается через спрос на экспортируемые отечественные продукты, а также через изменение объемов и цен экспорта. Кроме того, возможно снижение экспорта в связи с уменьшением объемов производства продуктов, что связано с карантином, но данный фактор нами не рассматривался. Учитывая непредсказуемость спроса на большинство экспортируемых позиций, остановимся в качестве примера только на нефти, мировая цена которой достаточно сильно реагирует на последствия пандемии.

Общее влияние изменений экспорта на ВВП можно разделить на первичное и вторичное, прямое и косвенное. Прямое влияние оценивается снижением валовой добавленной стоимости как реакцией на снижение экспорта в одной и той же отрасли. Снижаются объемы продаж, вследствие чего снижается выпуск, который определяет снижение ВДС отрасли с учетом коэффициентов прямых затрат. Косвенное влияние означает снижение выпуска и ВДС других отраслей под воздействием снижения спроса на исследуемую отрасль. Его величина определяется коэффициентами полных затрат той отрасли, спрос на товары или услуги которой падает. В обоих случаях рассмотрено первичное влияние экспорта на ВВП. Вторичное влияние осуществляется после перераспределения доходов между отраслями и 
секторами экономики. После снижения ВДС в отраслях снижаются доходы; в последующем спрос на внутреннем рынке снижается в результате снижения доходов секторов экономики. Таким образом, снижение экспортных доходов на первом этапе приводит в дальнейшем к снижению потребления, что ведет к уменьшению совокупного спроса на внутреннем рынке. Реакцией на снижение спроса является снижение выпуска и ВВП.

Если рассматривать в качестве основного фактора снижения экспортных доходов экономики биржевую цену нефти то, как правило, механизм влияния биржевых цен на макроэкономические показатели обычно описывается с помощью курса валют. В работе (Bykau, Ghodsi, Nezhadhossein, 2016) описана нелинейная регрессионная зависимость между ценой нефти Brent и курсом российского рубля к доллару, но приведенная модель работает лишь в условиях режима плавающего обменного курса рубля, установленного Центробанком, при отсутствии валютных интервенций. Отдаление реальных условий от модельных приводит к нарастающей погрешности в расчетах. И это неудивительно, поскольку любая регрессионная модель работает лишь в тех условиях, которые были характерны для периода наблюдения переменных модели. В 2020 г. эта модель уже не работала, в том числе в связи с некоторым изменением политики Центробанка, который в моменты резких спадов цены нефти поддерживал курс рубля валютными интервенциями.

Проблематичность использования курса рубля как функции от цены нефти в дальнейших расчетах обусловлена не только точностью регрессионных уравнений. Пересчет ВВП в доллары по номинальному обменному курсу возможен, но не будет отражать динамику реального экономического роста. Такой подход в целом противоречит общепринятой в России финансовой концепции дедолларизации, результатом которой стала отвязка внутренних цен на большинство товаров и услуг от курса доллара.

Безусловно, курс доллара не может не влиять на рост российской экономики, но это влияние может быть описано не одним, а по крайней мере тремя показателями, их соотношением. Наряду с девальвацией рубля сле- дует рассматривать уровень инфляции, а также рост или снижение денежного предложения (денежной массы). Если рост денежной массы опережает инфляцию, имеет место результативная стимулирующая рост ВВП политика Центробанка. Если инфляция превышает разницу между ростом денежной массы и ростом реального ВВП, имеет место ограничивающая политика, либо стимулирование не привело к должным результатам (вызвало всплеск инфляции, который привел к снижению реального ВВП). Данные подходы к анализу денежно-кредитной политики как факторов экономического роста, основанного на спросе, применены на примере белорусской экономики (Быков, Шаблинская, 2020).

С учетом вышеописанных допущений, базовый расчет ${ }^{4}$ влияния нефтяных цен на ВВП проведем в рублях в постоянных ценах. Предполагается, что чистые доходы от экспорта будут меняться пропорционально доходам, полученным от экспорта нефти, которые измеряются в долларах. Ocтальная часть экспортных доходов, не связанная прямо с нефтью, не меняется.

$$
X_{1}=X_{0} \cdot\left[1+\left(\frac{O P_{1}}{O P_{0}}-1\right) \cdot \frac{V A X_{-} P}{V A X}\right],
$$

где $X_{1}$ - чистые доходы от экспорта в прогнозном периоде, руб., без учета изменения курса (добавленная стоимость национального происхождения в экспорте);

$X_{0}$ - чистые доходы от экспорта в базовом периоде, руб.,

$O P_{1}$ - цена нефти Brent прогнозная, долл/барр.;

$O P_{0}$ - цена нефти Brent в отчетном периоде (на начало прогнозирования), долл/барр.;

$V A X \_P$ - добавленная стоимость, полученная от экспорта нефти и нефтепродуктов, с учетом наценок, руб.;

$V A X$ - добавленная стоимость национального происхождения в экспорте, с учетом наценок, руб.

Показатель $V A X \_P$ рассчитан на основе коэффициентов полных затрат, поэтому учи-

${ }^{4}$ Отметим, что в формулах (15) и (16) не используется матричная запись в соответствии с моделью Леонтьева, поэтому их следует рассматривать обособленно от предыдущих формул в плане математических обозначений. 
тывает прямое и косвенное влияние отрасли на изменение ВДС других отраслей.

Вторичное влияние снижения экспорта происходит через распределение доходов и снижение совокупного спроса на внутреннем рынке. Совокупный спрос (конечное использование за вычетом экспорта) $D$ уменьшается на величину $\left(X_{1}-X_{0}\right)$ после того, как секторы экономики (домашние хозяйства, фирмы, государство) уменьшат потребление в связи с уменьшением дохода. Если не учитывать, какую долю дохода потеряет каждый сектор, какие именно продукты в какой степени будет потреблять каждый сектор, а также не учитывать лаг времени между получением и использованием доходов, то снижение добавленной стоимости на внутреннем рынке в результате снижения доходов от экспорта определяется по формуле:

$$
\begin{aligned}
& V A D_{1}=\left(D+\left(X_{1}-X_{0}\right) \cdot\left(1-\frac{N B}{X \$}\right)\right) . \\
& \cdot\left(\left(1-\frac{M F P}{D}\right) \cdot \frac{V A X}{X}+\frac{T A X}{D}\right),
\end{aligned}
$$

где $V A D_{1}$ - добавленная стоимость, полученная за счет продаж отечественных и импортных товаров на внутреннем рынке, после изменений экспорта, руб. (добавленная стоимость от продаж отечественных продуктов на внутреннем рынке);

$D$ - спрос - конечное потребление и валовое накопление отечественных и импортных продуктов, руб.;

$N B$ - чистое кредитование по платежному балансу, в среднем, долл. США; США;

$X \$$ - экспорт, долл.

$X$ - экспорт, руб;

$M F P$ - импортные продукты в конечном потреблении и валовом накоплении;

$T A X$ - сумма налогов на конечное потребление и валовое накопление отечественных и импортных продуктов.
Первый коэффициент $\left(X_{1}-X_{0}\right) \cdot\left(1-\frac{N B}{X \$}\right)$ показывает уменьшение внутреннего спроса как вторичный эффект от снижения экспортных доходов секторов экономики. Он несколько меньше абсолютного снижения экспорта при наличии устойчиво профицитного торгового сальдо, потому что вслед за снижением экспорта вначале снижается отток капитала за границу, или чистое кредитование частного сектора. Второй коэффициент $\left(1-\frac{M F P}{D}\right)$ отражает изменение спроса на отечественную продукцию как долю от общего изменения спроса. Третий коэффициент $\frac{V A X}{X}$ характеризует добавленную стоимость отечественной экспортируемой продукции как долю от общего объема экспорта. Налоги $\frac{T A X}{D}$ также являются доходами, но облагаются ими не только отечественные, но и импортные продукты.

\section{Оценка ВВП в зависимости от цены нефти}

Фактические и прогнозные изменения компонентов ВВП - добавленной стоимости национального происхождения в экспорте $(V A X)$ и добавленной стоимости, создаваемой за счет продаж на внутреннем рынке $(V A D),-$ в ответ на изменения цены нефти представлены на рис. 4. По горизон-

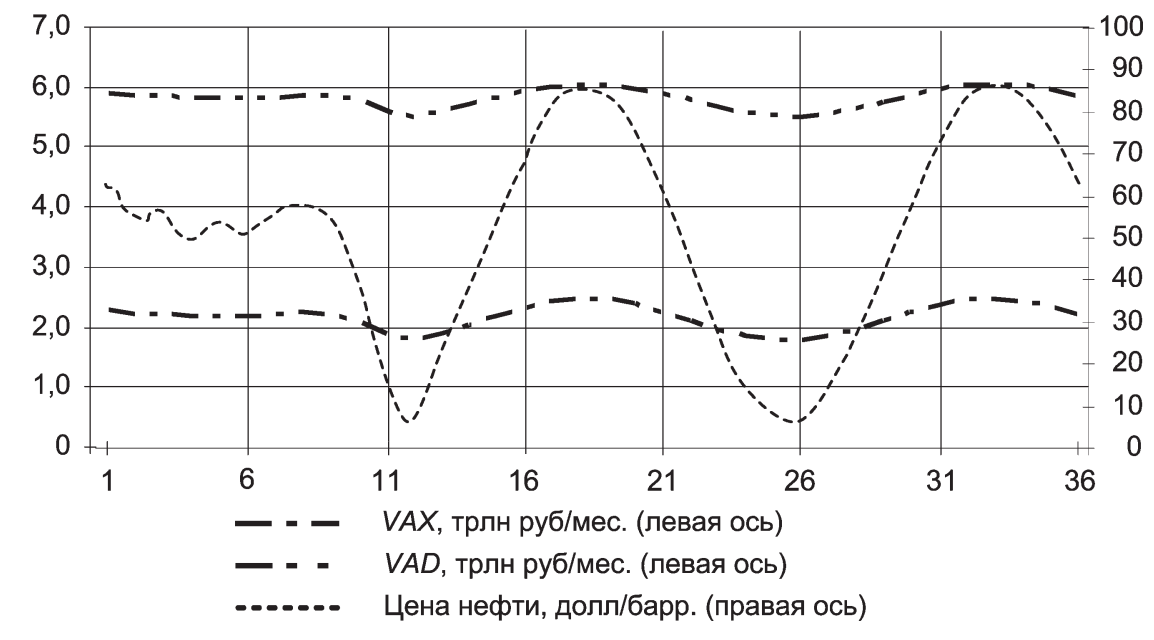

Рис. 4. Расчетные изменения составляющих ВВП как результат наблюдаемых и прогнозных колебаний цены нефти, трлн руб/мес.

Источник. Авторская разработка. 
тальной шкале обозначены месяцы, которые с 1-го по 12-ый соответствуют периоду с мая 2019 г. по апрель 2020 г., в расчетах использованы фактические данные по цене нефти за этот период. Месяцы с 13-го по 36-й отображают прогнозный период на два последующих года, до мая 2022 г. Прогнозная динамика цены нефти носит иллюстративный характер, с целью продемонстрировать возможную амплитуду колебаний данного показателя.

Следующий рисунок показывает эластичность компонентов ВВП по цене нефти - на сколько процентов изменится $V A X, V A D$ и ВВП в целом при изменении цены нефти на заданный процент.

В целом коэффициент эластичности ВВП по цене нефти равен 0,163. А при снижении цены нефти на 10 долл/барр. ВВП должен снизиться на 2,7\%. Первичный эффект снижения ВВП за счет сокращения добавленной стоимости в экспорте составит $1,5 \%$, вторичный эффект за счет снижения производства, вызванного снижением спроса на внутреннем рынке после сокращения экспортных доходов, составит $1,2 \%$ к ВВП. Полученные цифры велики и не слишком реалистичны. В публикациях на эту тему фигурируют цифры:

в 2014 г. эффект составил $-0,8 \%$ ВВП на каждые 10 долл/барр. снижения цены нефти ${ }^{5}$;

в 2020 г. каждое снижение стоимости барреля нефти на 10 долл. стоит ВВП России 0,5 п. п. роста ${ }^{6}$.

Реальные данные учитывают адаптацию экономики к изменению цены нефти, чего в модели нет. Например, зависимость курса рубля от цены нефти в долларах обеспечивает фактически неизменную величину рублевых доходов от экспорта нефти при широком диапазоне изменения биржевых котировок. Рублевые цены на отечественные товары и услуги, которые продаются на внутреннем рынке, также не сильно зависят от курса доллара.

Весьма существенное влияние на рост белорусской экономики оказывала денеж-

${ }^{5}$ URL: https://www.finam.ru/analysis/forecasts/ chuvstvitelnost-rossii-k-cene-na-neft-mify-i-realnost-20141111$1531 /$

${ }^{6}$ URL: http://www.finmarket.ru/shares/analytics/ $5190609 ? \mathrm{nt}=0$ но-кредитная политика, которая была описана двумя параметрами - изменением курса рубля к доллару и приростом денежной массы (Быков, Шаблинская, 2020). В российской экономике, которая в меньшей степени зависит от экспорта и импорта, денежно-кредитная политика должна рассматриваться весьма существенным фактором предотвращения экономического спада при негативных внешних шоках.

Чтобы не привязываться к доллару при анализе российской экономики возьмем параметры прироста денежной массы и инфляции. Например, в 2019 г. прирост денежной массы в национальном определении М2 составил $9,6 \%{ }^{7}$; за этот период ВВП вырос на 1,3\%; индекс-дефлятор ВВП составил $3,4 \%{ }^{8}$. Потенциальный прирост ВВП за счет денежного предложения определяется разницей между ростом денежного предложения, фактическим ростом реального ВВП и инфляцией, и может составить около 4,9\%. То есть, вполне возможно, что при ограничении денежного предложения в 2019 г. в российской экономике произошел бы экономический спад.

Предположим, в 2020 г., как и в предыдущем, потенциал прироста ВВП за счет денежного предложения составит 4,9\%. В этом случае негативное влияние снижения экспорта на ВВП может быть нейтрализовано за счет монетарных факторов. В каких именно пределах? На графике (рис. 5) линия изменения ВВП в зависимости от изменения цены нефти пересекается с горизонтальной линией потенциала денежного стимулирования в точке, соответствующей $30 \%$ снижению цены нефти. Это означает, что такое снижение (с 60 до 40 долл/ барр.) может и не привести к спаду вообще, если экономику будут стимулировать за счет увеличения эмиссии при ограничении инфляции. При данных допущениях и при среднегодовой цене ниже 40 долл/барр. спад все же начнется.

Представленные расчеты весьма условны, т. к. реальные пределы денежного стимулирования, при превышении которых начнется инфляция, рассчитать непросто,

${ }^{7}$ URL: http://www.cbr.ru/vfs/statistics/credit_statistics/ broadmoney_sa.xlsx

${ }^{8}$ URL: https://gks.ru/folder/313/document/812 
Влияние COVID-19 на российскую экономику: методологические подходы к оценке
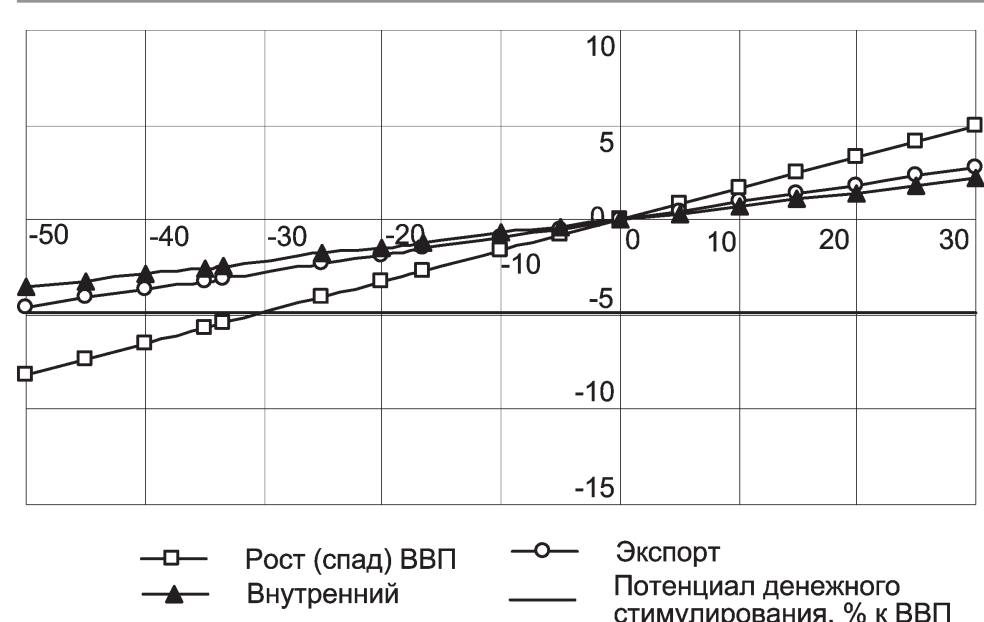

Рис. 5. Эластичность $V A X, V A D$ и ВВП по цене нефти снижения экспорта. Эти эффекты, будучи негативными, не обязательно должны суммироваться. Например, если движение капиталов ограничено в условиях карантина, можно еще смягчить монетарную политику, а также уменьшить импорт и отток капитала. Стимулирование роста в США и Европе может вызвать девальвацию доллара и евро и впоследствии привести в росту сырьевых цен. Пока данные сценарии маловероятны, но их нельзя исключать.

Источник. Авторская разработка.

и в этом случае следует говорить о вероятностных величинах. Например, при снижении цены нефти на $50 \%$, с 60 до 30 долл/ барр., без стимулирования экономика сократилась бы на 8,2\%, из которых первичный эффект в виде снижения добавленной стоимости экспорта составил бы 4,6\%, а вторичный - в виде последующего снижения спроса на отечественные товары и услуги в связи со снижением доходов секторов экономики составил бы 3,6\%. Однако при введении мер стимулирования можно увеличить ВВП на $4,9 \%$, и в этом случае реальный спад составит $3,3 \%$. В первом приближении мы получим потерю ВВП в размере 1,1\% на каждые 10 долл. уменьшения цены нефти.

При оценке общего влияния снижения цены нефти на ВВП будем исходить из среднегодовой цены в 40 долл/барр., а эффект от стимулирования установим в размере 4,9\% ВВП. Согласно рис. 5, снижение среднегодовой цены нефти с 60 до 40 долл/ барр. приведет к первичному снижению ВВП на 3\% и ко вторичному снижению на $2,4 \%$. С учетом эффекта стимулирования, спад ВВП составит 0,5\%.

Что касается прочих статей российского экспорта, включая природный газ, металлы, транспортные услуги, прогноз на год дать достаточно сложно ввиду слабой предсказуемости мировых рынков в текущих условиях.

Для уточнения этих прогнозов необходимо совмещение эффектов карантина и
Пандемия COVID-19 может рассматриваться как внешний шок для всей мировой экономики, приведший к значительным экономическим последствиям. Неопределенность относительно возобновления нормального функционирования и экономического роста в будущем высока. Неопределенность связана не только непосредственно с эпидемиологическим процессом, но и с возможной реакцией потребителей на продолжение или смягчение карантинных мер, а также действий правительств и центральных банков.

Представляется, что в таких условиях экономисты могут давать лишь приближенные оценки с последующей их корректировкой и уточнением. Это, однако, не означает, что подобные оценки бесполезны или носят сугубо теоретический характер. Чем раньше будет ставится задача прогнозирования динамики макроэкономических параметров национальной экономики под воздействием негативных эффектов пандемии, тем более обоснованной и продуманной будет экономическая политика по стимулированию возобновления экономического роста в будущем.

Осуществленные выше расчеты на базе системы таблиц «затраты - выпуск» для российской экономики были направлены на оценку двух ключевых факторов воздействия COVID-19 на некоторые макроэкономические параметры.

1. Карантинные меры, которые привели к серьезному снижению активности населения, их мобильности и потребительс- 
ких расходов, что напрямую сказалось на объеме внутреннего спроса.

2. Конъюнктура мировых товарных рынков, в первую очередь, рынка нефти, которая ввиду высокой неопределенности, вызванной пандемией, подвержена турбулентности. Данный фактор будет воздействовать на макроэкономические параметры национальной экономики посредством снижения экспортных доходов.

Подстановка прогнозируемых значений конечного внутреннего спроса по всем 98 отраслям (продуктам), выделяемым в российской системе таблиц «затраты выпуск», в модифицированное уравнение межотраслевого баланса Леонтьева позволила оценить как общее снижение ВВП в 2020 г. на уровне 4,33\%, так и удельный вклад разных секторов экономики в это снижение (наибольший вклад - 79\% - обеспечивает сектор услуг, что в общем и целом вполне ожидаемо).

Оценка влияния второго фактора осуществлялась на основе укрупненных пропорций между отдельными показателями российской экономики, которые также были рассчитаны с использованием данных таблиц «затраты - выпуск», но без непосредственного использования аппарата модели Леонтьева. При этом учитывался и такой вторичный эффект, как уменьшение внутреннего спроса вследствие снижения экспортных доходов секторов экономики, учитывалась также доля спроса на отечественную продукцию в общем изменении спроса и добавленная стоимость отечественной экспортируемой продукции в общем объеме экспорта.

Расчеты показывают, что влияние экспорта на ВВП в значительной мере опосредуется денежно-кредитной политикой. В качестве базового сценария стоит исходить из среднегодовой цены 40 долл/барр. при эффекте от денежного стимулирования в 4,9\%. В этом случае спад ВВП за счет изменения экспорта нефти составит 0,5\%.

В проведенных расчетах остались неучтенными некоторые параметры - в частности, возможное изменение экспортных доходов по остальным экспортным позициям, кроме нефти и нефтепродуктов. Цены на некоторые из них (природный газ, металлы) могут снизиться вслед за ценами на нефть. Динамика цен на сельхозпродукцию сейчас непредсказуема. В конечном итоге, объем валового экспорта по продуктам, за исключением нефти и нефтепродуктов, нами оставлен без изменения, но предложенные подходы позволяют исследователям самостоятельно рассчитать влияние экспорта данных продуктов на изменение ВВП.

В качестве обобщающего вывода обосновывается оценка совместного влияния эффектов карантина и снижения цены нефти на уровне около минус 5\% ВВП. Несмотря на вполне ожидаемую условность такой оценки, она может служить некоторым ориентиром в планировании стимулирующей экономической политики и базой для последующих более точных оценок.

\section{СПИСОК ЛИТЕРАТУРЫ (REFERENCES)}

Быков А.А., Шаблинская Т.В. 2020. Декомпозиция факторов экономического роста, основанного на спросе, с применением методологии межотраслевого баланса. Белорусский экономический журнал. № 1. С. 4-21. [Bykau A., Shablinskaya T. 2020. Decomposition of demand-driven economic growth factors using input-output methodology. Belorusskiy ekonomicheskiy zhurnal. No 1. PP. 4-21. (In Russ.)]

Готовский А.В. 2019. Обеспечение сбалансированного экономического роста Республики Беларусь. Белорусский экономический журнал. № 2. C. 4-24. [Gotovsky A. 2019. Ensuring a balanced economic growth of the Republic of Belarus. Belorusskiy ekonomicheskiy zhurnal. No 2. PP. 4-24. (In Russ.)]

Кравцов М.К., Антаневич А.А. 2015. Прогнозирование важнейших показателей белорусской экономики на основе балансово-оптимизационной модели. Белорусский экономический журнал. № 1. C. 110-123. [Kravtsov M., Antanevich A. Forecasting main indicators of Belarus's economy based on balanceoptimization model. Belorusskiy ekonomicheskiy zhumal. No 1. PP. 110-123. (In Russ.)]

Точицкая И. 2019. Оценка добавленной стоимости в экспорте Беларуси. Рабочий материал Исследовательского центра ИПМ WP/19/03. Минск: Исследовательский центр ИПМ. С. 3. [Tochitskaya I. 2019. Assessment of the value added in exports of Belarus. Rabochiy material Issledovatel'skogo tsentra IPM WP/19/03. Minsk: Issledovatel'skiy tsentr IPM. P. 1-15. (In Russ.)] 
Bykau A., Ghodsi A., Nezhadhossein H. 2016. Impact of oil prices on Russian ruble on condition of floating exchange rate regime. The 5th International Virtual Scientific Conference on Informatics and Management Sciences. PP. 67-69. DOI: 10.18638/ ictic.2016.5.1.274.

Chen S., Igan D., Pierri N., Presbitero A. 2020. The economic impact of Covid-19 in Europe and the US: Outbreaks and individual behaviour matter a great deal, non-pharmaceutical interventions matter less. VOX CEPR Policy Portal. URL: https://voxeu.org/article/ economic-impact-covid-19-europe-and-us

Chen X., Cheng L.K., Fung K.C., Lau L.J. 2004. The estimation of domestic value-added and employment induced by exports: An application to Chinese exports to the United States. Stanford University Working Paper. URL: https:// www.researchgate.net/publication/255575337 The Estimation of Domestic_Value-Added and Employment_Induced_by_Exports_An_Application_ to_Chinese_Exports_to_the_United__States
Friston K.J., Parr T., Zeidman P., Razi A., Flandin G., Daunizeau J., Hulme O.J., Billig A.J., Litvak V., Moran R.J., Price C.J., Lambert C. 2020. Dynamic casual modelling of COVID-19. Technical report. URL: https://arxiv.org/ftp/arxiv/ papers/2004/2004.04463.pdf

Koopman R.B., Wang Z., Wei S.-J. 2008. How Much of Chinese Exports is Really Made in China? Assessing Foreign and Domestic Value-added in Gross Exports. NBER Working Paper No 14109. URL: https://pdfs.semanticscholar.org/e594/ ea6d01cafe6f0018423a3541f6b1b7e0e150.pdf?_ga= 2.90326170.164791978.1591791689-1790891446. 1576842253

Leontief W. 1986. Input-output economics. New York; Oxford: Oxford University Press.

Wood R. 2011. Construction, stability and predictability of an input-output time-series for Australia. Economic Systems Research. Vol. 23. No 2. PP. 175-211.

In citation: Belorusskiy Economicheskiy zhurnal. 2020. No 2. PP. 25-37.

Belarusian Economic Journal. 2020. No 2. PP. 25-37.

\title{
INFLUENCE OF COVID-19 ON THE RUSSIAN ECONOMY: METHODOLOGICAL APPROACHES TO THE ASSESSMENT BASED ON THE INPUT-OUTPUT TABLES
}

\author{
Aliaksei Bykau', Uladzimir Parkhimenka², Sergey Tolkachev ${ }^{3}$ \\ Authors affiliation: ${ }^{1}$ Belarusian State Economic University (Minsk, Belarus); \\ ${ }^{2}$ Belarusian State University of Informatics and Radioelectronics (Minsk, Belarus); \\ ${ }^{3}$ Financial University under the Government of the Russian Federation (Moscow, \\ Russia).
}

Corresponding author: Aliaksei Bykau (aliaksei.bykau@yandex.ru).

ABSTRACT. We made an attempt to evaluate the impact of the COVID-19 pandemic on the economy of Russian Federation, based on estimates of domestic demand decrease for each of the 98 industries (products) identified in the system of input-output tables. The analysis was carried out in the context of identifying two key factors of demand changes: lockdown measures that directly limit domestic demand, as well as global oil prices, leading to a reduction in export income of the economy and a subsequent reduction in demand for goods and services in the domestic market. The obtained quantitative estimates are presented in the context of selected industries, sectors and the Russian economy as a whole. As a generalized conclusion, it justifies the assessment of the joint effect of lockdown and a decrease in oil exports at about $5 \%$ of GDP.

KEYWORDS: «input - output» analysis, economic growth, economic impact of the COVID-19 pandemic, lockdown, Russian Federation.

JEL-code: C67, D57, E17, O41.

DOI: $10.46782 / 1818-4510-2020-2-25-37$ 